\title{
Lifetime Model Development for Integration in Power Management of HEVs by Terms of Minimizing Fuel Consumption and Battery Degradation
}

\author{
Nejra Beganovic ${ }^{1}$, Bedatri Moulik ${ }^{2}$, Ahmed Mohamed Ali $^{3}$, and Dirk Söffker ${ }^{3}$ \\ 1 Faculty of Science, Technology and Media \\ Department of Electronics Design, Mid-Sweden University \\ Holmgatan 10, 85170 Sundsvall, Sweden \\ nejra.beganovic@miun.se \\ ${ }^{2}$ Amity School of Engineering and Technology \\ Department of Electrical and Electronics Engineering, Amity University \\ Amity Campus Sector 125, 201303 Noida, India \\ bmoulik@amity.edu \\ ${ }^{3}$ Faculty of Engineering \\ Chair of Dynamics and Control, University of Duisburg-Essen \\ Lotharstraße 1-21, 47057 Duisburg, Germany \\ ahmed.ali@uni-due.de \\ soeffker@uni-due.de
}

\begin{abstract}
Along with increasingly frequent use of electric and hybrid electric vehicles, the constraints and demands placed on the them become stricter. The most noticeable challenge considering Hybrid Electric Vehicles (HEVs) is to provide an optimal power flow between multiple electric sources alongside provided as less as possible aging of energy storage components. To provide efficient battery usage with respect to batteries lifetime, it becomes unavoidable to develop battery lifetime models, which do not only reflect the State-of-Heath (SoH) but also allow battery lifetime prediction. The lifetimeoriented battery models have to be integrated in power management. To be used efficiently and to provide optimal power split ensuring mitigation of battery degradation without sacrificing desired power consumption, accurate modeling of battery degradation is of utmost importance. This implies that gradual battery degradation, which is directly affected by applied loading profiles, has to be monitored and used as additional control input. Moreover, the lifetime model developed in this case has to provide model outputs also in the timeframe of power management. In this contribution, a machine state-based lifetime model for electric battery source is devel-

\footnotetext{
Nejra Beganovic et al. This is an open-access article distributed under the terms of the Creative Commons Attribution 3.0 United States License, which permits unrestricted use, distribution, and reproduction in any medium, provided the original author and source are credited.
}

oped. In this particular case, different degradation states as well as machine state transitions are identified in accordance to current operating conditions. Here, the change in charging/discharging rate (C-rate), overcharging/undercharging of the battery (depth-of-discharge), and the temperature are taken in consideration to define machine model states. The End-of-Lifetime (EoL) is defined as deviation between nominal and current ampere-hour (Ah)-throughput. The proposed machine state-based lifetime model is verified based on existing battery lifetime models using simulation setup. The developed lifetime model in this way serves as a prerequisite for its integration into power management with an aim to provide the trade-off between aforementioned conflicting objectives; fuel consumption and battery degradation.

\section{INTRODUCTION}

An evident scarcity of oil supplies alongside increased negative impact on environment concerning pollution, emission of greenhouse gases, and its corresponding contribution to global warming emerge the development and introduction of a number of improvements in transportation sector (Serrao, Onori, \& Rizzoni, 2011; Marano, Onori, Guezennec, Rizzoni, \& Madella, 2009). Due to increased human population, consequently higher number of automobiles, a viable solution for energy efficient, environmental-friendly, and low- 
cost transportation led to an utilization of Hybrid Electric Vehicles (HEV) and Plug-in Hybrid Electric Vehicles (PHEV) to a higher extent than in previous years. Although hybrid electric vehicles are characterized by greater complexity, consequently also by increased costs, a number of advantages in comparison to conventional vehicles, such as reduced fuel consumption through hybridization and mitigated polluting emissions, cause still unsolved issues in this field to become increasingly interesting and thoroughly discussed in recent years (Onori, Spagnol, Marano, Guezennec, \& Rizzoni, 2012).

Total energy required for vehicle propulsion in case of $\mathrm{HEV} / \mathrm{PHEV}$ is often split among fuel energy and electrical energy source(s). As such, efficient power management is indisputable by terms of providing optimal solution with respect to available energy resources, component aging, drive profile, and traffic conditions (Onori, Serrao, \& Rizzoni, 2016; Sabri, Danapalasingam, \& Rahmat, 2016). The optimization objective as such is defined over minimization of fuel consumption and mitigation/minimization of aging of energy sources with retained desired power demand. The optimization of fuel consumption concerning integration of Lithium-ion Battery (LIB) aging model is treated as additional cost of optimization quantified using appropriate lifetime model. In this contribution, the LIB lifetime model for integration into power management is developed. Model development serves as a prerequisite for its further integration. Machine state-based LIB lifetime model is developed concerning a number of degradation indicators of LIB occurred during twenty drive cycles. The model is verified using simulation setup.

The contribution based on exiting data models is organized as follows: i) after introduction in Section 1, current state-of-art concerning power management and quantification of battery aging is given in Section 2, ii) machine state-based battery lifetime model is detailed in Section 3, iii) followed by presentation and discussion of obtained results in Section 4. The contribution closes with conclusion and outlook in Section 5.

\section{State-OF-ART}

\subsection{Powertrain Configurations and Power Management Strategies}

As conventional vehicles with Internal Combustion Engines (ICA) are replaced by HEV/PHEV/EV, the leverage on power management and control strategies assigned within it becomes larger (Sabri et al., 2016; Bayindir, Gözüküçük, \& Teke, 2011). By incorporating a number of different energy sources in HEV/PHEV vehicles, power management becomes crutial to control energy flows between the different sources and sinks. Taking into account limited energy from electrical energy sources, goals defined through minimizing fuel consumption and aging of system components while maintaining the same vehicle performance, different $\mathrm{HEV} / \mathrm{PHEV}$ configurations are discussed in a number of contributions (Sabri et al., 2016; Peng, He, \& Xiong, 2017; Vora et al., 2018; Wang, Ma, \& Wang, 2018). In dependence of most common HEV/PHEV powertrain configurations (series, parallel, or series-parallel configuration), different power management strategies for providing optimal power flow between energy sources are developed. Still unsolved challenge from this perspective is an inclusion of an additional cost into power management objective function related to the aging of energy source components (primarily LIB battery packs as rechargeable electrical sources). In this case, the development of an accurate lifetime model of LIB/LIB battery packs is crucial, as they have direct impact on the efficiency of power management and overall system costs.

Improving fuel economy of PHEVs using rule-based energy management algorithms and Dynamic Programming (DP) due to simplicity of implementation and fast computation are commonly used based on multi-objective optimization (Peng et al., 2017). In (Peng et al., 2017), an optimization-based rule development procedure is explained and validated by Hardware-in-Loop (HIL) simulation experiments, but information about battery degradation is not taken in consideration. Also in (Zhang \& Xiong, 2015) hierarchical control strategy for ensuring optimal power split between different energy sources of PHEV without consideration of LIB lifetime is proposed. Control strategy here consists of two layers, whereas the upper layer regulates the engine-generator and hybrid energy-storage system whilst the lower layer regulates the power split between the battery and ultracapacitor. A driving pattern recognition is integrated in adaptive energy management through classifying typical driving cycles into different driving patterns. Dynamic programming is employed to develop optimal control strategies for different driving patterns (Zhang \& Xiong, 2015). Further, power management with integrated degradation of the battery and consequent impact on fuel consumption and overall system cost is discussed in (Vora et al., 2018). In the aforementioned contribution, a powertrain simulation model and battery degradation model are used to conduct a lifecycle economic analysis such as payback period and rate of return. According to obtained results, it becomes noticeable that the fuel consumption increases by $10 \%$ during the overall service lifetime. Proposed framework thus enables system cost analysis through integration of an impact of battery degradation and replacement on the total cost. Moreover, the battery is recognized as the most expensive component (Vora et al., 2018). Similarly, relatively short battery longevity affecting at the same time an overall price of the system is discussed in (Wang et al., 2018). An energy management strategy for power split, aiming not only to reduce fuel consumption but also to prolong battery lifetime, is performed in order that the instantaneous battery usage is penalized by its influence on the battery lifetime. By terms of 
battery degradation, the influence is quantified as the batteryfading index corresponding to aging rate in dependence of operating conditions. Here, the multi-objective optimization problem is achieved using model predictive control (Wang et al., 2018).

The trend of improvements and advances to be introduced in this field are noticeable from the discussion in Section 2 but inevitably include the integration of Remaining Useful Lifetime (RUL) of battery in power management. The aim is to extend the lifetime of overall system, reduce costs without impacting system performance, and additionally to adapt the power management rules to actual SoH of the battery. Accordingly, the development of accurate LIB lifetime model for an integration into power management and ensuring battery longevity is of main concern in this contribution, as depicted in Figure 2.

\subsection{Battery Aging}

Numerous battery degradation models are presented in literature, most of them focusing on single technology (Marano et al., 2009; Onori et al., 2012; Jafari, Khan, \& Gauchia, 2018; Lipu et al., 2018; Lin, Hao, Liu, \& Jia, 2018; Suri \& Onori, 2016; Song, Hofmann, Li, Han, \& Ouyang, 2015). Characterized by specific key variables to be monitored and integrated within the mathematical presentation of the model, existing models can be discussed either from material level or application level (Jafari et al., 2018). Here, stochastic processes related to the aging of LIB are discussed thoroughly as the LIBs are commonly used in HEV/PHEV vehicles as energy source. Factors that show a huge impact on battery aging are primarily battery charging/discharging rate, Depth-of-Discharge (DoD), and temperature. Higher battery charging/discharging rates as well as higer temperature contribute to faster capacity fading, as same as lower DoD. Due to increased temperature as well as high charging/discharging rates and the DoD, the complex electrochemical reactions occurring in the battery are accelerated, causing the growth of the solid electrolyte interphase layer and increasing the internal resistance of the battery. Consequently, the capacity of a battery becomes reduced. All of these three quantities are affected by loading profiles; here drive cycle/pattern (Jafari et al., 2018; Lipu et al., 2018; Lin et al., 2018). The battery EoL by these terms is quantified using capacity fade or an increase of internal resistance as they are directly related. The most commonly accepted definition of battery aging is taken as a reduction in capacity to $80 \%$ of its nominal value. The accelerated aging process through fast charging, beside the fact that this drastically reduce battery lifetime, can have impacts on battery safety.

Comprehensive review of remaining useful lifetime estimation methods for LIB can be found in (Lipu et al., 2018). It becomes noticeable that the modeling approaches can be grouped as follows: i) measurement assessment approach, ii) adaptive approach, and iii) data-driven approach. The Coulomb counting method, requiring occasional recalibration and iterative complete charging/discharging of the battery is one possible way of correlating State-of-Health (SoH) to DoD. Alongside Coulomb counting method, open circuit voltage method and impedance spectroscopy are employed to measure intermediate system variables (such as charging/discharging currents, voltage, and/or temperature) and correlate them to SoH/degradation state of the battery. Adaptive approaches like Kalman filtering, particle filterbased methods, or least-square methods belong to the second group and mostly introduce some uncertainties into the models. The largest group according to (Lipu et al., 2018) is the group of data-driven methods based on the analysis of a huge amount of captured data. Utilizing fuzzy-logic methods, support vector machines, some form of artificial neural networks, and similar methods, underlying electrochemical processes within these modeling approaches do not need to be known in advance. Alongside battery lifetime modeling approaches, a health conscious fast charging frameworks are developed to reduce the charge duration alongside battery degradation (Lin et al., 2018). For instance, an electrolyte enhanced single particle model with degradation mechanisms and a multi-objective optimal control problem is discussed in (Lin et al., 2018). Battery lifetime model is employed along with DP technique to find the optimal charging strategies, concerning charging time and battery degradation. The analysis in (Lin et al., 2018) is relied neither on real drive pattern nor adapted power management but traditional constant current/constant voltage approach and health conscious fast charging framework. Contrary, a semi-empirical battery aging model identified using the data representing mimicking loading conditions that HEV/PHEV battery encounters in real driving scenarios is formulated in (Suri \& Onori, 2016). The introduced aging model is used to construct so-called severity factor map, which characterizes gradual degradation of the battery under different operating conditions. The developed battery lifetime model is further used to integrate a battery degradation criterion within a multi-objective optimization, aiming to provide battery aging as well as fuel consumption minimization. The model in (Suri \& Onori, 2016) is validated by simulations conducted using a hybrid electric vehicle simulator, whereas it is proven that the severity factor map relied aging model can be effectively used to predict and control battery degradation. Similarly, lifetime model developed for integration in power management is elaborated in (Marano et al., 2009; Onori et al., 2012). A generic approach to LIB lifetime modeling applicable to a number of vehicle classes based on the concept of accumulated charge throughput (Ahthroughput) under real drive profile is proposed in aforementioned contribution. The assumption lying behind is that the damage is accumulated proportionally to charge transfer in or from the battery, modulated by a severity factor associ- 
ated with the operating conditions. The most critical issue reported in (Marano et al., 2009; Onori et al., 2012) is to quantify the topology of severity map with respect to battery sizing and powertrain control with concerned battery damage accumulation model.

This contribution focuses to battery lifetime modeling for integration in power management also to provide optimal/intelligent power split between energy sources in multisource HEVs/PHEVs. It is of high importance to develop LIB lifetime model which would overtake existing shortcomings of existing models, especially concerning real life drive profiles and the necessity for a priori knowledge about underlying processes of LIB aging. The lifetime model proposed in this contribution requires neither a priori knowledge about physical processes occurring in LIB nor high computational complexity (including complex analytical relations). It is of high importance to note that the developed model can be used to estimate State-of-Health at some time point in future based on applied operating conditions and available measurements. Taking the information about predicted $\mathrm{SoH}$ in future, it becomes possible to affect the lifetime by adapting power management strategy, as depicted in Figure 1. In Figure 1, three different loading profiles/operating conditions with accompanying lifetimes are depicted, each of them showing different level of accumulated damage and used lifetime predicted from $t_{\text {now }}$.

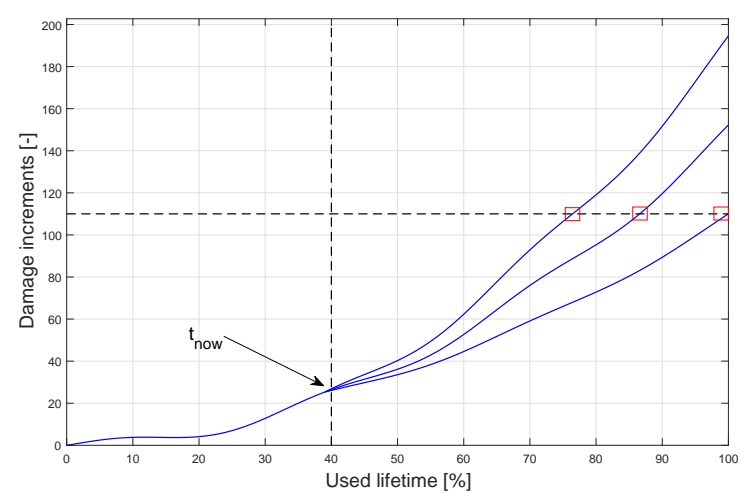

Figure 1. Integration of current health state of the system into power management.

\section{BATTERY LIFETIME MODELING}

Battery data model proposed in this contribution is machine state-based lifetime model, tending to describe LIB aging by reflecting particular system inputs to predefined machine states in accordance to current operating conditions, as initially introduced in (Beganovic \& Söffker, 2017). The number of considered states is three, whereas each state is represented by a mathematical equation with different state-based optimized parameters. In this contribution, the model and results obtained in (Onori et al., 2012) and (Song et al., 2015)
Table 1. Model states.

\begin{tabular}{l|l}
\hline \hline State & Expression \\
\hline \hline$S_{1}$ & $Q_{\text {loss }}=A_{1} e^{-\frac{B_{1}+C_{1} \cdot C_{\text {rate }}}{R \cdot T}} \cdot\left(A h_{\text {throughput }}\right)^{z_{1}}$ \\
\hline$S_{2}$ & $Q_{\text {loss }}=A_{2} e^{-\frac{B_{2}+C_{2} \cdot C_{\text {rate }}}{R \cdot T}} \cdot\left(A h_{\text {throughput }}\right)^{z_{2}}$ \\
\hline$S_{3}$ & $Q_{\text {loss }}=A_{3} e^{-\frac{B_{3}+C_{3} \cdot C_{\text {rate }}}{R \cdot T}} \cdot\left(A h_{\text {throughput }}\right)^{z_{3}}$ \\
\hline
\end{tabular}

are used for the own modeling procedure and parameter definition. In a later context, the literature data can be easily replaced by experimental data.

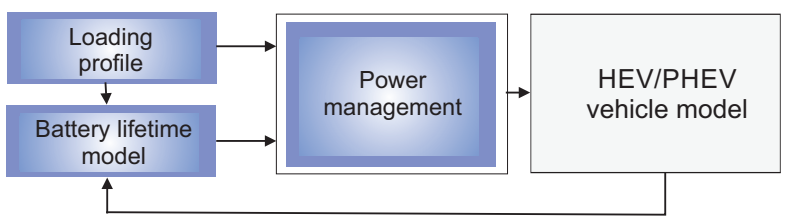

Figure 2. Overall power management scheme with integrated degradation model of LIB.

Inputs to the lifetime model are charging/discharging LIB current, DoD (defined as $D o D=100-S o C$ expressed in $\%$ ), and the temperature, as is shown in Figure 3. In this case, the temperature and DoD are used to quantify the impact of temperature on $A h_{\text {throughput }}$ using severity factor map, as described in detail in (Onori et al., 2012). The Ahthroughput is further correlated to battery capacity loss and used lifetime. Concretely, battery capacity loss is expressed in (Song et al., 2015) as

$$
Q_{\text {loss }}=A e^{-\frac{B+C \cdot C_{\text {rate }}}{R \cdot T}} \cdot\left(A h_{\text {throughput }}\right)^{z},
$$

where $A, B, C$, and $z$ are constants, $T$ is the temperature in kelvins, $A h_{\text {throughput }}$ is the current throughput, and $R$ is the gas constant. Mathematical equation 1 is used in this contribution alongside severity factor map for $A h_{\text {throughput }}$ calculation and lifetime estimation. The $C_{\text {rate }}$ is considered as $1 C$. The parameters $A, B, C$, and $z$ are varied in dependence of operating conditions, whereas each of aforementioned parameter is assigned to particular machine state. Three different machine states are identified according to different operating conditions, namely $S_{1}, S_{2}$, and $S_{3}$. Here, $S_{1}$ is the state with the most favorable operating conditions from the aspect of degradation, and $S_{3}$ is the state with the most unfavorable operating conditions from the aspect of degradation. The states are described as given in Table 1. The model thus has four parameters per state which has to be optimized. In total, twelve model parameters are optimized using Nondominated Sorting Genetic Algorithm (NSGA-II).

State transitions are defined in correspondence to input variable threshold exceedance and their rates of change, as is shown in Figure 4. In Figure 4, rate of change of Ahthroughput is stated as $I_{D I F F}$ and is used to define state 


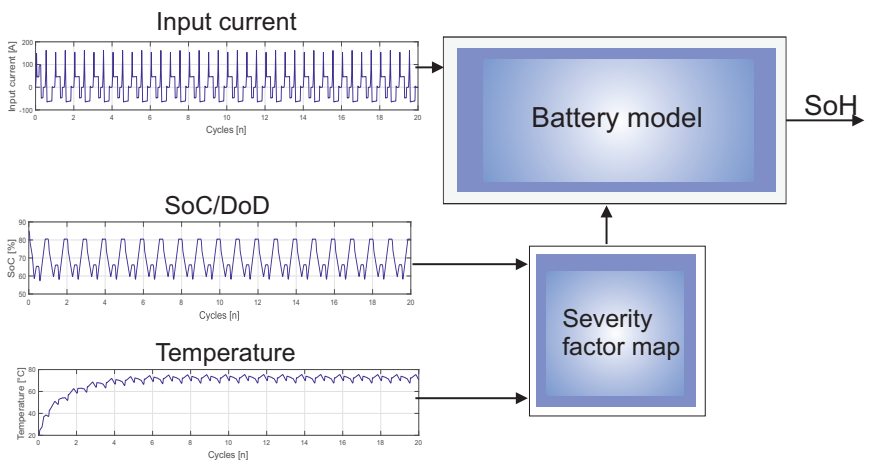

Figure 3. Degradation model of LIB and model inputs.

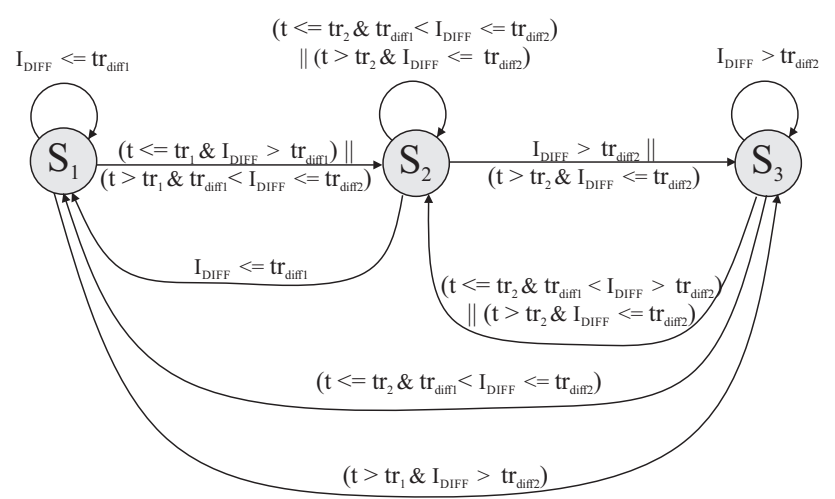

Figure 4. Model states and state transitions.

transitions. It can be seen from Figure 4 that the transition from $S_{1}$ to $S_{2}$ occur either when the temperature exceeds predefined threshold $t r_{1}$ whilst $I_{D I F F}$ lies between $t r_{\text {diff } 1}$ and $t r_{\text {diff } 2}$ or when the temperature is lower than $t r_{1}$ but $I_{D I F F}$ exceeds $t r_{\text {diff } 1}$. Similarly, state transition from $S_{2}$ to $S_{3}$ occur either when $I_{D I F F}$ becomes higher than $t r_{d i f f 2}$ or when the temperature exceeds predefined threshold $t r_{2}$ and $I_{D I F F}$ lies below $t r_{\text {diff } 2}$. State transition from $S_{1}$ to $S_{3}$ occur only when the temperature is found below $t r_{1}$ and $I_{D I F F}$ is greater than $t r_{\text {diff } 2}$. The transition from $S_{3}$ to $S_{1}$ occur when the temperature drops to predefined threshold $t r_{2}$ simultaneously with $I_{D I F F}$ is found between $t r_{d i f f 1}$

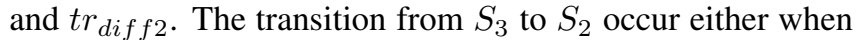
the temperature drops to predefined threshold $t r_{2}$ simultaneously when $I_{D I F F}$ equals to the value between $t r_{\operatorname{diff} 1}$ and $t r_{\text {diff } 2}$ or when the temperature becomes higher than $t r_{2}$ with $I_{D I F F}$ dropped below $t r_{\text {diff } 1}$. The state from $S_{2}$ is changed to $S_{1}$ when $I_{D I F F}$ drops to $t r_{\text {diff } 1 \text {. As such, the }}$ state is not changed as long as the change in $I_{D I F F}$ and temperature lies within predefined thresholds. This preknowledge is qualitatively effecting the structure of the model by defined by the state machine model provided. The related parameters of the state machine variables have to be defined with a suitable data-driven-based training.
Table 2. Optimized parameters.

\begin{tabular}{l|l|l|l|}
\hline \hline Parameters & State $S_{1}$ & State $S_{2}$ & State $S_{3}$ \\
\hline \hline$A_{x}$ & 0.004 & 0.0044 & 0.0054 \\
\hline$B_{x}$ & 324.1979 & 439.479 & 1000.0 \\
\hline$C_{x}$ & 15863.0 & 15657.0 & 17557.0 \\
\hline$z_{x}$ & 0.6619 & 0.0546 & 0.1773 \\
\hline
\end{tabular}

\section{RESULTS AND DISCUSSION OF RESULTS}

\subsection{Battery Lifetime Model}

The inputs of LIB lifetime model are shown in Figure 5. In the first diagram, vehicle speed over twenty drive cycles used for analysis is depicted. As already stated, input current (consequently its change over time) and the DoD/SoC are considered as model inputs. In addition, the impact of temperature on battery aging is analyzed by integrating temperature measure into the model over severity factor map.
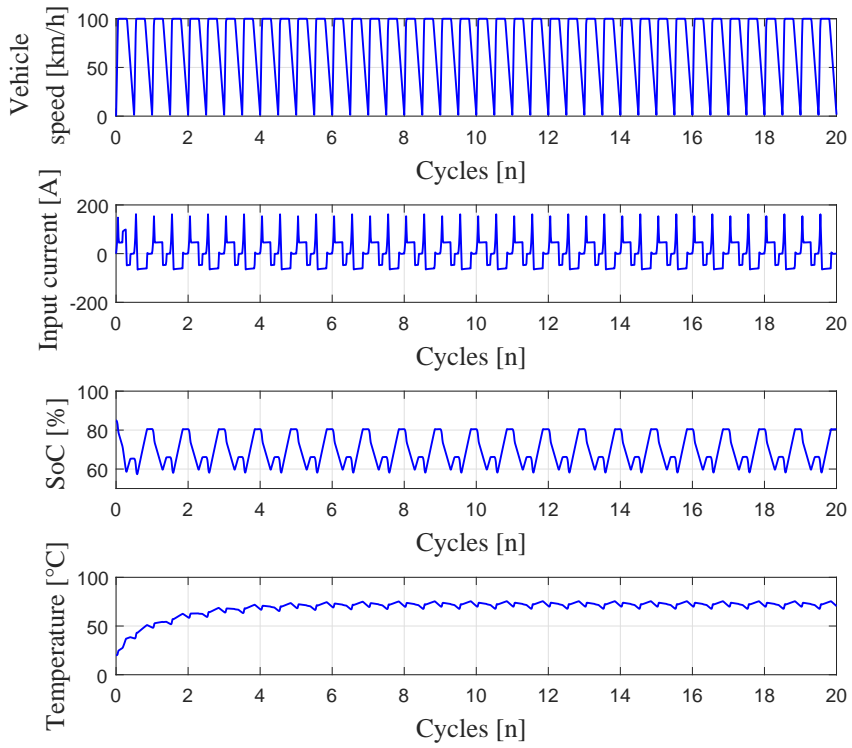

Figure 5. Input variables to LIB lifetime model.

Optimized model parameters for each state using NSGA-II are given in Table 2 . The parameters are defined by terms of guaranteed lowest estimation error by optimization when compared with reference values, implying the objective function given as

$$
\operatorname{obj}(t)=\mid \operatorname{reference}(t)-\text { actual value }(t) \mid \text {. }
$$

An estimation of capacity loss, which can directly be corre- 
Table 3. Prediction error.

\begin{tabular}{l|l|l|}
\hline \hline Prediction point & End-point value & Percentage error \\
\hline \hline $20 \%$ & 4698.2 & $100-93.964=6.036$ \\
\hline $40 \%$ & 4653.3 & $100-93.066=6.934$ \\
\hline $60 \%$ & 4701.1 & $100-94.022=5.978$ \\
\hline $80 \%$ & 4980.2 & $100-99.604=0.396$ \\
\hline
\end{tabular}

lated either to used or remaining lifetime, as well as Endof-Lifetime prediction using proposed machine state-based model is verified using simulation setup. Obtained results are given in Figure 6. Figure 6 depicts battery capacity loss during twenty drive cycles. Aforementioned cycle is defined as a change is $\mathrm{SoC}$ from 58 to $83 \%$ (the third diagram in Figure 6).

Obtained reference capacity drop during twenty drive cycles (step-wise behavior, black line) and estimated capacity loss obtained using proposed machine state-based model, both expressed in percentages are shown in the first diagram in Figure 6. As the EoL is considered as capacity loss of $20 \%$, it implies that with the capacity drop of $20 \%$ the battery is neither capable to endure additional loading anymore nor to provide safe use. The second diagram shows absolute error and its propagation over time, whilst machine states used for calculation of $\Delta Q_{\text {loss }}$ are shown in the last diagram of Figure 6. As only twenty drive cycles are analyzed here, capacity drop is a function of twenty drive cycles showing very low absolute error and acceptable level of congruence with reference lifetime values. This means, accumulated $\Delta Q_{\text {loss }}$ during twenty drive cycles in this particular case equals to 0.08 . By extending obtained results to additional drive cycles, concerning constant capacity loss rate and EoL detected when accumulated absolute value of $\Delta Q_{\text {loss }}$ equals to twenty, the battery is feasible to withstand

$$
\frac{20 \text { drive cycles }}{0.08 \text { capacity loss per } 20 \text { drive cycles }}=250
$$

in total $250 \cdot 20=5000$ drive cycles until its EoL as example.

\subsection{Prediction of Battery Lifetime}

To extend obtained results to additional drive cycles and to predict battery lifetime, a few assumptions are considered: i) the points in time used for prediction are varied by $20 \%$, ii) no further change of operating conditions after considered time point $t_{\text {now }}$ is assumed, and iii) a lifetime is affected in the same manner by each drive cycle after $t_{\text {now }}$. Stated $t_{\text {now }}$ is the time point at which used LIB reaches $20,40,60$, and $80 \%$ of its lifetime. Taking in consideration stated assumptions and developed battery lifetime model, obtained results are depicted in Figure 7.

In the first diagram of Figure 7, reference and estimated ca-
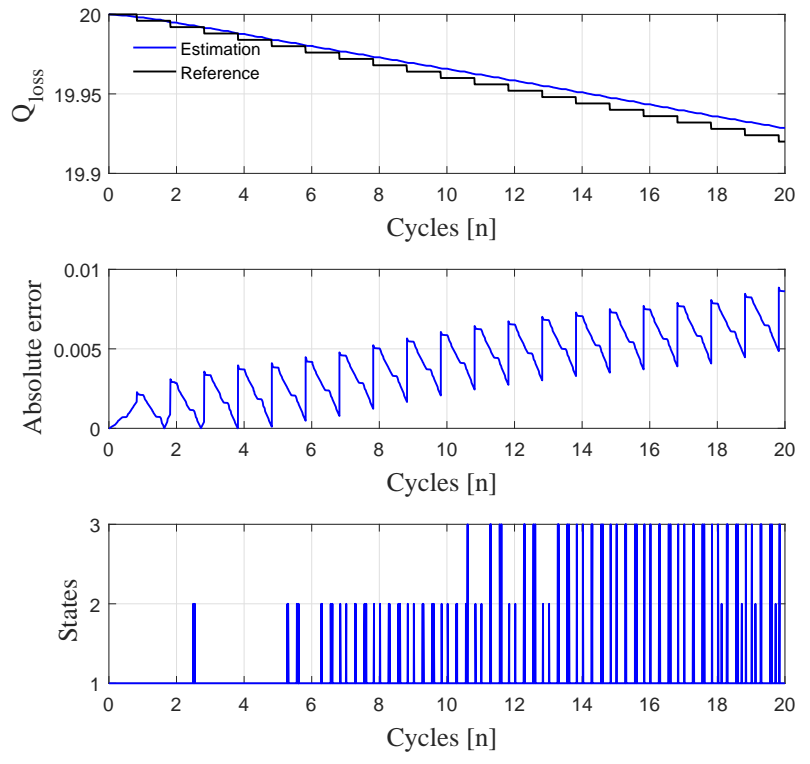

Figure 6. Obtained results using proposed machine statebased lifetime model.

pacity drop when only $20 \%$ of battery service lifetime is known is shown. When expressed in terms of drive cycles, it equals to $5000 \cdot 0.2=2000$. The rest of $80 \%$ of battery service lifetime is predicted. Similarly, in the second, third, and fourth diagram of Figure 7, reference and estimated capacity drop when 40,60 , and $80 \%$ of battery service lifetime is known is depicted. The rest of 60,40 , and $20 \%$ of battery service lifetime, respectively, is predicted. At first glance, it can be seen that the deviation between reference and estimated values is low in all considered cases. It implies that the model is capable to predict the lifetime of battery (battery capacity drop) over time with acceptable error, which as the largest amounts almost $7 \%$ as calculated in Table 3.

\section{Conclusion AND OUTLOOK}

In this contribution, a lifetime-oriented machine state-based battery model for integration into power management is developed with an aim to provide an optimal power flow between multiple electric sources alongside provided as less as possible aging of battery/battery packs.

The proposed model in contrast to most other models does not require a priori knowledge about underlying electrochemical processes occurring in LIB. It includes simplified analytical relations connecting operating conditions and LIB aging. Accordingly, gradual battery degradation explained through proposed model can be used as additional control input to provide optimal power flow. Different degradation states as well as machine state transitions are identified in accordance to current operating conditions. According to the results, it can 

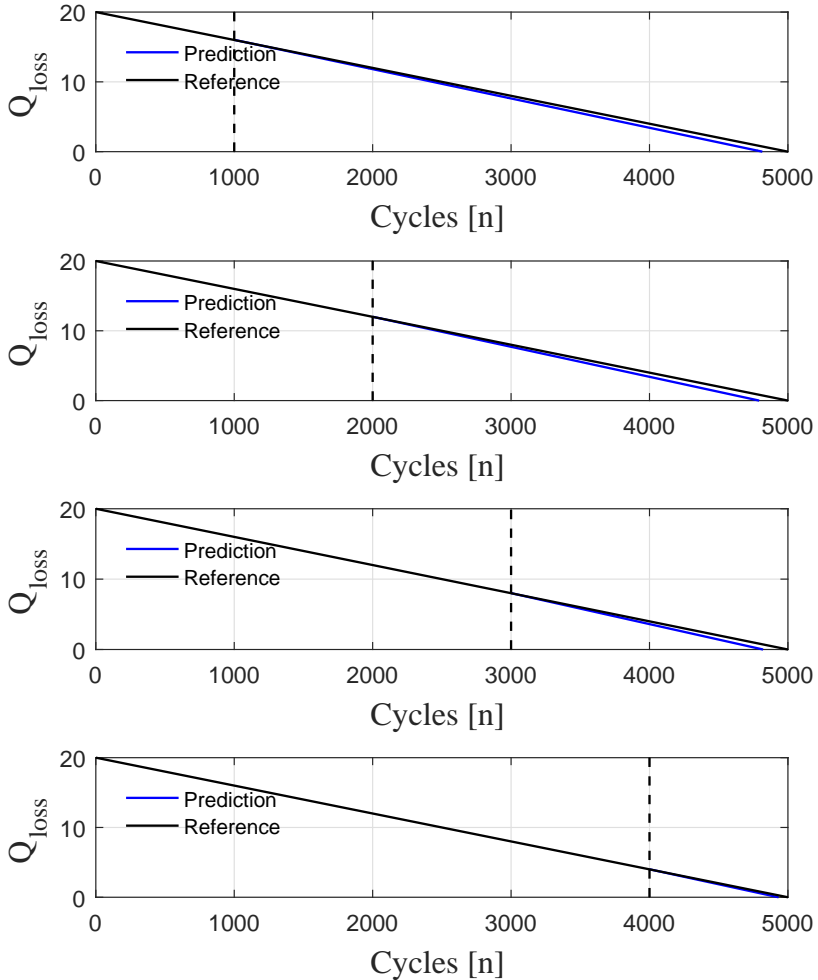

Figure 7. Obtained results using proposed machine statebased lifetime model.

be shown that absolute error lie below $0.01 \%$ when twenty drive cycles are considered, and the prediction error using the same model applied to extended timespan equals to almost $7 \%$ in the worst case, when $80 \%$ of lifetime is predicted and only $20 \%$ of lifetime is a priori known. As such, the model proposed in this contribution is ideal for integration into power management, including control with integrated information about predicted battery lifetime.

The improvement in proposed model related to experimental model validation and severity factor map adjustment concerning particular battery/battery packs can be considered for future work.

\section{NOMENCLATURE}

$I C A \quad$ Internal Combustion Engines

$H E V \quad$ Hybrid Electric Vehicle

$P H E V \quad$ Plug-in Hybrid Electric Vehicles

EoL End-of-Lifetime

$L I B \quad$ Lithium-ion Battery

$S o C \quad$ State-of-Charge

DoD Depth-of-Discharge

SoH State-of-Health
$D P \quad$ Dynamic Programming

HIL Hardware-in-Loop

\section{REFERENCES}

Bayindir, K. Ç., Gözüküçük, M. A., \& Teke, A. (2011). A comprehensive overview of hybrid electric vehicle: Powertrain configurations, powertrain control techniques and electronic control units. Energy Conversion and Management, 52(2), 1305-1313.

Beganovic, N., \& Söffker, D. (2017). Remaining lifetime modeling using state-of-health estimation. Mechanical Systems and Signal Processing, 92, 107-123.

Jafari, M., Khan, K., \& Gauchia, L. (2018). Deterministic models of li-ion battery aging: It is a matter of scale. Journal of Energy Storage, 20, 67-77.

Lin, X., Hao, X., Liu, Z., \& Jia, W. (2018). Health conscious fast charging of li-ion batteries via a single particle model with aging mechanisms. Journal of Power Sources, 400, 305-316.

Lipu, M. H., Hannan, M., Hussain, A., Hoque, M., Ker, P. J., Saad, M., \& Ayob, A. (2018). A review of state of health and remaining useful life estimation methods for lithium-ion battery in electric vehicles: Challenges and recommendations. Journal of Cleaner Production, 205, 115-133.

Marano, V., Onori, S., Guezennec, Y., Rizzoni, G., \& Madella, N. (2009). Lithium-ion batteries life estimation for plug-in hybrid electric vehicles. In 2009 IEEE Vehicle Power and Propulsion Conference (pp. 536-543).

Onori, S., Serrao, L., \& Rizzoni, G. (2016). Hybrid electric vehicles: Energy management strategies. Springer.

Onori, S., Spagnol, P., Marano, V., Guezennec, Y., \& Rizzoni, G. (2012). A new life estimation method for lithiumion batteries in plug-in hybrid electric vehicles applications. International Journal of Power Electronics, 4(3), 302-319.

Peng, J., He, H., \& Xiong, R. (2017). Rule based energy management strategy for a series-parallel plug-in hybrid electric bus optimized by dynamic programming. Applied Energy, 185, 1633-1643.

Sabri, M., Danapalasingam, K., \& Rahmat, M. (2016). A review on hybrid electric vehicles architecture and energy management strategies. Renewable and Sustainable Energy Reviews, 53, 1433-1442.

Serrao, L., Onori, S., \& Rizzoni, G. (2011). A comparative analysis of energy management strategies for hybrid electric vehicles. Journal of Dynamic Systems, Measurement, and Control, 133(3), 031012.

Song, Z., Hofmann, H., Li, J., Han, X., \& Ouyang, M. (2015). Optimization for a hybrid energy storage system in electric vehicles using dynamic programing approach. Applied Energy, 151-162. 
Suri, G., \& Onori, S. (2016). A control-oriented cycle-life model for hybrid electric vehicle lithium-ion batteries. Energy, 96, 644-653.

Vora, A. P., Jin, X., Hoshing, V., Shaver, G., Varigonda, S., \& Tyner, W. E. (2018). Integrating battery degradation in a cost of ownership framework for hybrid electric vehicle design optimization. Proc. of the Institution of Mechanical Engineers, Part D: Journal of Automobile Engineering, 0954407018802663.

Wang, X., Ma, S., \& Wang, J. (2018). Predictive energy management for hybrid electric vehicles considering extension of the battery life. Proc. of the Institution of Mechanical Engineers, Part D: Journal of Automobile Engineering, 232(4), 499-510.

Zhang, S., \& Xiong, R. (2015). Adaptive energy management of a plug-in hybrid electric vehicle based on driving pattern recognition and dynamic programming. $A p-$ plied Energy, 155, 68-78.

\section{BIOGRAPHIES}

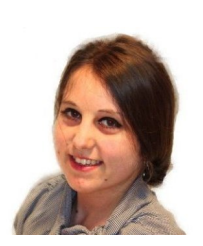

Nejra Beganovic received the B.Sc. and M.Sc. in electrical engineering from the University of Sarajevo, Chair of Automatic Control and Electronics, BosniaHerzegovina, in 2009 and 2011 respectively. In 2016, she has received the Dr.-Ing. degree from the University of Duisburg-Essen, Engineering Faculty, Germany. From October 2017 to February 2019, she was an Assistant Professor at the International Burch University, Sarajevo. Since February 2019, she is a postdoctoral researcher at Mid-Sweden University, Department of Electronics Design, Sweden. Her research interests include structural health monitoring, lifetime prognostics, and advanced signal processing methods with special emphasis on real-time implementation of classification and prognostic algorithms.

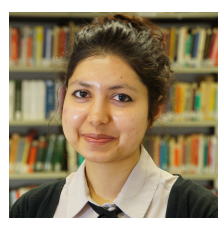

Bedatri Moulik received her Bachelors' degree in Electrical Engineering and Masters' degree in Control and Instrumentation Engineering from West Bengal University of Technology, Kolkata, India. She received her Dr.-Ing. Degree from the Engineering Faculty, University of Duisburg-Esssen, Germany. She is now an Assistant Professor at Amity University, Noida, India in the department of Electrical and Electronics Engineering.

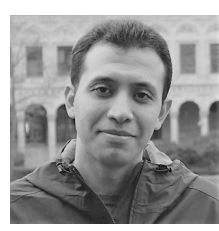

Ahmed M. Ali received the B.Sc. degree and the M.Sc. degree, both in Mechanical Engineering from the Military Technical College, Cairo, Egypt, in 2008 and 2015 respectively. He is now with the Chair of Dynamics and Control, University of Duisburg-Essen, where he works towards the Ph.D. degree in the field of optimal design and control of hybrid vehicles.

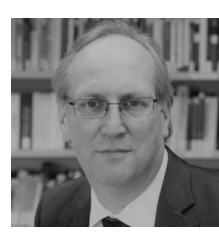

Dirk Söffker received the Dr.-Ing. degree in mechanical engineering and the Habilitation degree in automatic control/safety engineering from University of Wuppertal, Wuppertal, Germany, in 1995 and 2001, respectively. Since 2001, he leads the Chair of Dynamics and Control with the Engineering Faculty, University of Duisburg-Essen, Germany. His current research interests include elastic mechanical structures, modern methods of control theory, human interaction with safety systems, safety and reliability control engineering of technical systems, and cognitive technical systems. 